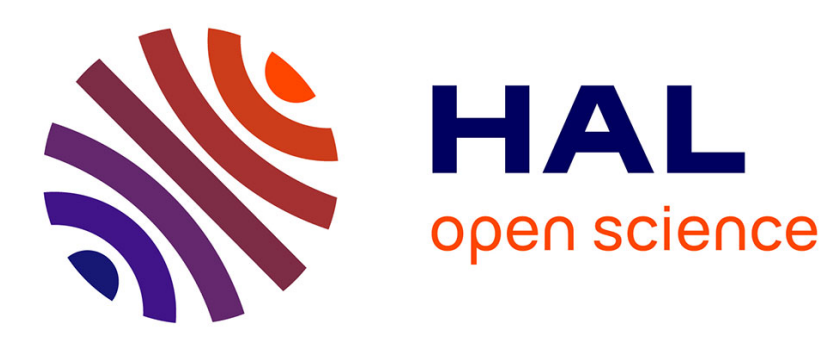

\title{
Observability of linear systems with commensurate delays and unknown inputs
}

Francisco Javier Bejarano, Gang Zheng

\section{To cite this version:}

Francisco Javier Bejarano, Gang Zheng. Observability of linear systems with commensurate delays and unknown inputs. Automatica, 2014, 50 (8), pp.2077-2083. 10.1016/j.automatica.2014.05.032 . hal-00967944

\section{HAL Id: hal-00967944 \\ https://inria.hal.science/hal-00967944}

Submitted on 8 Mar 2017

HAL is a multi-disciplinary open access archive for the deposit and dissemination of scientific research documents, whether they are published or not. The documents may come from teaching and research institutions in France or abroad, or from public or private research centers.
L'archive ouverte pluridisciplinaire HAL, est destinée au dépôt et à la diffusion de documents scientifiques de niveau recherche, publiés ou non, émanant des établissements d'enseignement et de recherche français ou étrangers, des laboratoires publics ou privés. 


\title{
Observability of Linear Systems with Commensurable Delays Unknown Inputs ${ }^{\text {光 }}$
}

\author{
Francisco Javier Bejarano ${ }^{\mathrm{a}}$, Gang Zheng ${ }^{\mathrm{b}}$ \\ ${ }^{a}$ SEPI, ESIME Ticomán, IPN, Av. San José Ticomán 600, C.P. 07340, Mexico City, Mexico. \\ ${ }^{b}$ Non-A, INRIA - Lille Nord Europe, 40 avenue Halley, Villeneuve d'Ascq 59650, France.
}

\begin{abstract}
This papers investigates the observability analysis for linear time systems whose outputs are affected by unknown inputs. Three different definitions of observability are proposed. By introducing the Smith form and comparing the invariant factors, sufficient condition is deduced for each proposed definition of observability. Several examples are given for the purpose of highlighting the effectiveness of the proposed methods.
\end{abstract}

Key words: Delay systems, commensurate delays, observability, unknown inputs.

\section{Introduction}

Time delay systems are widely used to model many concrete applications, like chemical and biological process and many results have been published to treat this kind of systems for different aspects Richard (2003); Sename (2001). The analysis of observation for time delay systems can be dated back to the 80's of the last century Lee and Olbrot (1981); Olbrot (1981); Salamon (1980); Rabah (1995). For this issue, different definitions of observability have been proposed, such as strong observability, spectral observability and weak observability.

For linear time delay systems, various aspects of the observability problem have been studied in the literature, using different methods such as the functional analytic approach Bhat and Koivo (1976) or the algebraic approach Brewer et al. (1986); Fliess and Mounier (1998); Sontag (1976).

\footnotetext{
Corresponding author F.J. Bejarano

Email addresses: javbejarano@yahoo.com.mx (Francisco Javier Bejarano), gang.zheng@inria.fr (Gang Zheng)
}

Preprint submitted to Elsevier

April 8, 2014 
For nonlinear time delay systems, by using the theory of non-commutative rings Moog et al. (2000), the observability problem has been studied in Xia et al. (2002) for systems with known inputs, and in Zheng et al. (2011) for systems with unknown inputs. The associated observer for some classes of time delay systems can be found in Conte et al. (2003); Sename (2001); Darouach (2006); Fattouh et al. (1999); Fu et al. (2004) and the references therein.

Nonetheless, the majority of the existing works on observability analysis are focused on time delay systems whose outputs are not affected by unknown inputs. However, this situation might exist in some practical applications and this motivates the work of this paper. Here, we deal with time delay systems which are linear and whose delays are commensurable. We consider that delay may appear in the state, input, and output The aim is searching for conditions allowing for the reconstruction of the entire state vector using backward, actual, and forward output information.

The contributions of this paper are as follows. Firstly, we introduce the Unknown Input Observability (UIO), backward UIO and forward UIO concepts. For each one of the proposed observability definitions, we obtain sufficient conditions that can be verified by using some matrices depending on the original system parameters. The established condition for the unknown input observability turns out to be a generalization of the already known condition for systems with unknown inputs, but without delays (in that case such condition is also a necessary one), and also it is a generalization of the known strongly observable condition for linear systems with commensurable delays, but without unknown inputs. Due to the methodology used along the paper, the results may be applied to systems over polynomial rings.

The following notations will be used: $\mathbb{R}$ is the field of real numbers, $\mathbb{R}_{\neq 0}$ is the set of nonzero real numbers. The set of nonnegative integers is denoted by $\mathbb{N}_{0} . \mathbb{R}[\delta]$ is the polynomial ring over the field $\mathbb{R}$. The Laurent polynomial ring is denoted as $\mathbb{R}\left[\boldsymbol{\delta}, \boldsymbol{\delta}^{-1}\right] . \mathbb{R}^{n}[\boldsymbol{\delta}]$ is the $\mathbb{R}[\boldsymbol{\delta}]$-module whose elements are the vectors of dimension $n$ and whose entries are polynomials. By $\mathbb{R}^{q \times s}[\delta]$ we denote the set of matrices of dimension $q \times s$, whose entries are in the $\mathbb{R}[\delta]$. For $f(\delta)$, a polynomial of $\mathbb{R}[\delta], \operatorname{deg} f(\boldsymbol{\delta})$ is the degree of $f(\boldsymbol{\delta})$. For a matrix $M(\delta), \operatorname{deg} M(\delta)$ (the degree of $M(\delta)$ ) is defined as the maximum degree of all the entries $m_{i j}(\delta)$ of $M(\delta) . \operatorname{det} M(\delta)$ is the determinant of this matrix, and $\operatorname{rank} M(\delta)$ means the rank of the matrix $M(\delta)$ over $\mathbb{R}[\delta]$. The acronym for greatest common divisor is $\mathrm{gcd}$. 


\section{Formulation of the Problem and Definitions}

We will deal with the following class of linear systems with commensurate delays

$$
\begin{aligned}
& \dot{x}(t)=\sum_{i=0}^{k_{a}} A_{i} x(t-i h)+\sum_{i=0}^{k_{b}} B_{i} w(t-i h) \\
& y(t)=\sum_{i=0}^{k_{c}} C_{i} x(t-i h)+\sum_{i=0}^{k_{d}} D_{i} w(t-i h)
\end{aligned}
$$

where the state vector $x(t) \in \mathbb{R}^{n}$, the system output vector $y(t) \in \mathbb{R}^{p}$, and the unknown input vector $w(t) \in \mathbb{R}^{m}$, the initial condition $\varphi(t)$ is a piecewise continuous function $\varphi(t):[-k h, 0] \rightarrow \mathbb{R}^{n}$ $\left(k=\max \left\{k_{a}, k_{b}, k_{c}, k_{d}\right\}\right)$; thereby $x(t)=\varphi(t)$ on $[-k h, 0] . A_{i}, B_{i}$, and $C_{i}$ matrices are of appropriate dimension with entries belonging to $\mathbb{R}$. System (1) may be represented in a compact form, by using the delay operator (backward time-shift operator) $\delta: x(t) \rightarrow x(t-h)$. Thus, we have

$$
\begin{aligned}
& \dot{x}(t)=A(\delta) x(t)+B(\delta) w(t) \\
& y(t)=C(\delta) x(t)+D(\delta) w(t)
\end{aligned}
$$

where $A(\delta):=\sum_{i=0}^{k_{a}} A_{i} \delta^{i}, B(\delta):=\sum_{i=0}^{k_{b}} B_{i} \delta^{i}, C(\delta):=\sum_{i=0}^{k_{c}} C_{i} \delta^{i}$, and $D(\delta):=\sum_{i=0}^{k_{d}} D_{i} \delta^{i}$. Hence, every entry of these matrices belongs to the polynomial ring $\mathbb{R}[\delta]$. As for $x(t ; \varphi, w)$, we mean the solution of the delay differential equation of system (1) with the initial condition equal to $\varphi$, and the input vector equal to $w$. Analogously, we define $y(t ; \varphi, w):=C(\delta) x(t ; \varphi, w)+D(\delta) w(t)$, that is, to be the system output of (1) when $x(t)=x(t ; \varphi, w)$.

Practically, what we search for is to find conditions allowing us for estimating the state $x(t)$. In order to tackle the problem in a more formal way, we will use the following observability definitions.

Definition 1 (Unknown Input Observability). System (1) is called unknown input observable (UIO) on the interval $\left[t_{1}, t_{2}\right]$ iff there exist $t_{1}^{\prime}$ and $t_{2}^{\prime}\left(t_{1}^{\prime}<t_{2}^{\prime}\right)$ such that, for all input $w$ and every initial condition $\varphi$,

$$
y(t ; \varphi, w)=0 \text { for all } t \in\left[t_{1}^{\prime}, t_{2}^{\prime}\right] \text { implies } x(t ; \varphi, w)=0 \text { for all } t \in\left[t_{1}, t_{2}\right] .
$$

Definition 2 (Backward UIO). System (1) is said to be backward UIO (BUIO) on $\left[t_{1}, t_{2}\right]$ iff for all $\bar{t} \in\left[t_{1}, t_{2}\right]$ there exist $t_{1}^{\prime}<t_{2}^{\prime} \leq \bar{t}$ such that, for all input $w$ and every initial condition $\varphi$,

$$
y(t ; \varphi, w)=0 \text { for all } t \in\left[t_{1}^{\prime}, t_{2}^{\prime}\right] \text { implies } x(t ; \varphi, w)=0 \text { for all } t \in\left[t_{1}, t_{2}\right] .
$$


Definition 3 (Forward UIO). System (1) is said to be forward UIO (FUIO) on $\left[t_{1}, t_{2}\right]$ iff for all $\bar{t} \in\left[t_{1}, t_{2}\right]$ there exist $t_{2}^{\prime}>t_{1}^{\prime} \geq \bar{t}$ such that, for all input $w$ and every initial condition $\varphi$,

$$
y(t ; \varphi, w)=0 \text { for all } t \in\left[t_{1}^{\prime}, t_{2}^{\prime}\right] \text { implies } x(t ; \varphi, w)=0 \text { for all } t \in\left[t_{1}, t_{2}\right] .
$$

Remark 1. These definitions are essentially formulated following the observability definitions given in Kalman et al. (1969) for linear systems. Basically, Unknown Input Observability considers the case when the state vector can be reconstructed using past and future values of the system output. As for the backward UIO, it is related with the case when only actual and past values of the system output are needed for the state reconstruction. Finally, the forward UIO defines a property which theoretically allows for the reconstruction of the state vector using only actual and future values of the system output.

Obviously, either BUIO or FUIO implies UIO. Also, it should be noted that BUIO and FUIO observability do not exclude each other. Let us see for instance that the system

$$
\begin{aligned}
& \dot{x}_{1}=x_{2}, \quad \dot{x}_{2}=x_{1}+\delta x_{2} \\
& y_{1}=\delta x_{1}, \quad y_{2}=x_{2}
\end{aligned}
$$

is FUIO observable on $\left[t_{1}, t_{1}+h\right]\left(t_{1} \geq h\right)$, since $y(t)=0$ on $\left[\bar{t}, t_{1}+2 h\right]$ implies $x(\bar{t})=0$ for all $\bar{t} \in\left[t_{1}, t_{1}+h\right]$. And also it is BUIO observable on $\left[t_{1}, t_{1}+h\right]$ since $y(t)=0$ on $\left[t_{1}-h, \bar{t}\right]$ implies $x(\bar{t})=0$ for all $\bar{t} \in\left[t_{1}, t_{1}+h\right]$.

In the next section we will search for sufficient conditions allowing for the test of UIO property, which at the same time provide us of a constructive way to reconstruct $x(t)$ in finite time.

\section{Basic Results}

The study of the observability for linear systems (without delays) has been successfully tackled by using geometric methods, in particular invariant subspaces. For the time delay case such methods cannot be followed straightforwardly, however still many of those ideas can be borrowed (see Conte et al. (2003), Conte et al. (2007)). Here we will not follow strictly a geometric method, 
however, in its spirit the idea still comes from the results of geometric methods of standard linear systems, as we will see below.

Let $P(\delta)$ be a matrix of dimension $q \times s$ with rank equal to $r$ (clearly $r \leq \min \{q, s\}$ ). We know that there exists an invertible matrix $T(\delta)$ over $\mathbb{R}[\delta]$ (representing elementary row operations) such that $P(\delta)$ is put into the (column) Hermite form. Thus, we have that

$$
T(\delta) P(\delta)=\left[\begin{array}{c}
P_{1}(\delta) \\
0
\end{array}\right]
$$

where $P_{1}(\delta)$ dimension $r \times s$, and $\operatorname{rank} P_{1}=r$. Also, there exist two invertible matrices $U(\delta)$ and $V(\delta)$ over $\mathbb{R}[\delta]$ (representing elementary row and column operations, respectively) such that $P(\delta)$ is reduced to its Smith form, i.e.,

$$
U(\boldsymbol{\delta}) P(\boldsymbol{\delta}) V(\boldsymbol{\delta})=\left[\begin{array}{cc}
\operatorname{diag}\left(\psi_{1}(\boldsymbol{\delta}) \cdots \psi_{r}(\boldsymbol{\delta})\right) & 0 \\
0 & 0
\end{array}\right]
$$

where the $\left\{\psi_{i}(\delta)\right\}$ are monic nonzero polynomials satisfying

$$
\psi_{i}(\boldsymbol{\delta}) \mid \psi_{i+1}(\boldsymbol{\delta}) \text { and } d_{i}(\boldsymbol{\delta})=d_{i-1}(\boldsymbol{\delta}) \psi_{i}(\boldsymbol{\delta})
$$

where $d_{i}(\delta)$ is the gcd of all $i \times i$ minors of $P(\delta)\left(d_{0}=1\right)$. The $\left\{\psi_{i}(\delta)\right\}$ are called invariant factors, and $\left\{d_{i}(\delta)\right\}$ determinant divisors.

Following the ideas of Silverman (1969) and Molinari (1976), let us define $\left\{\Delta_{k}(\delta)\right\}$ matrices generated by the following algorithm,

$$
\begin{gathered}
\Delta_{0} \triangleq 0, G_{0}(\boldsymbol{\delta}) \triangleq C(\boldsymbol{\delta}), F_{0}(\boldsymbol{\delta}) \triangleq D(\boldsymbol{\delta}) \\
S_{k}(\boldsymbol{\delta}) \triangleq\left[\begin{array}{c}
\Delta_{k}(\boldsymbol{\delta}) B(\boldsymbol{\delta}) \\
F_{k}(\boldsymbol{\delta})
\end{array}\right], \quad k \geq 0 \\
{\left[\begin{array}{cc}
F_{k+1}(\boldsymbol{\delta}) & G_{k+1}(\boldsymbol{\delta}) \\
0 & \Delta_{k+1}(\boldsymbol{\delta})
\end{array}\right] \triangleq T_{k}(\boldsymbol{\delta})\left[\begin{array}{cc}
\Delta_{k}(\boldsymbol{\delta}) B(\boldsymbol{\delta}) & \Delta_{k}(\boldsymbol{\delta}) A(\boldsymbol{\delta}) \\
F_{k}(\boldsymbol{\delta}) & G_{k}(\boldsymbol{\delta})
\end{array}\right]}
\end{gathered}
$$

where $T_{k}(\delta)$ is an invertible matrix over $\mathbb{R}[\delta]$ that transforms $S_{k}$ into its Hermite form, and $\Delta_{0}$ is 
of dimension 1 by $n$. Then, $\left\{M_{k}(\delta)\right\}$ matrices are defined as follows,

$$
\begin{gathered}
M_{0}(\boldsymbol{\delta}) \triangleq N_{0}(\boldsymbol{\delta}) \triangleq \Delta_{0} \\
N_{k+1}(\boldsymbol{\delta}) \triangleq\left[\begin{array}{c}
N_{k}(\boldsymbol{\delta}) \\
\Delta_{k+1}(\boldsymbol{\delta})
\end{array}\right], \text { for } k \geq 0 \\
{\left[\begin{array}{c}
M_{k+1}(\boldsymbol{\delta}) \\
0
\end{array}\right] \triangleq\left[\begin{array}{cc}
\operatorname{diag}\left(\psi_{1}^{k+1}(\boldsymbol{\delta}), \ldots, \psi_{r_{k+1}+1}^{k+1}(\boldsymbol{\delta})\right) & 0 \\
0 & 0
\end{array}\right]=U_{k+1}(\boldsymbol{\delta}) N_{k+1}(\boldsymbol{\delta}) V_{k+1}(\boldsymbol{\delta})}
\end{gathered}
$$

with $U_{k}(\delta)$ and $V_{k}(\delta)$ being invertible matrices over $\mathbb{R}[\delta]$ that transform $N_{k}$ to its Smith form. It is worthy noting that, by construction, $F_{k}(\delta)$ and $M_{k}(\delta)$ matrices have both full row rank, and $M_{k}(\delta)$ has always $n$ columns.

Remark 2. $N_{k}$ is used in Silverman (1969) to calculate the null-output weakly unobservable subspace, denoted by $\mathscr{V}^{*}$, for linear (with no delays) systems with unknown inputs. It is known that the system (with no delays) is strongly observable (= UIO according to our definition) if, and only if, such a subspace contains only the zero vector.

However for delay system it is not the case, as can be seen by the simple example $\dot{x}=0$, $y=(1-\delta) x(t)$. In this case $\mathscr{V}^{*}=0$, but $y=0$, for any $x(t)$, which is constant along the time.

It is intuitively clear that since $\left\{T_{k}\right\},\left\{U_{k}\right\}$, and $\left\{V_{k}\right\}$ are invertible over $\mathbb{R}[\boldsymbol{\delta}]$, then the invariant factors of $\left\{M_{k}\right\}$ matrices should not depend on the particular selection of those former invertible matrices. This is true as we will prove it in the following lemma.

Lemma 1. $\left\{M_{k}\right\}$ matrices generated by (4)-(5) are invariant from the choice of $\left\{T_{k}\right\},\left\{U_{k}\right\}$, and $\left\{V_{k}\right\}$.

Proof. Firstly, it is easy to verify that for two matrices $\Delta_{1}$ and $\bar{\Delta}_{1}$ calculated in (4) with two different matrices $T_{0}$ and $\bar{T}_{0}$, respectively, we obtain that

$$
\bar{\Delta}_{1}=J_{1} \Delta_{1}
$$

for some invertible matrix $J_{1}$ over $\mathbb{R}[\delta]$. Indeed, since $\bar{T}_{0}$ and $T_{0}^{-1}$ are invertible over $\mathbb{R}[\boldsymbol{\delta}]$ and $F_{1}$ has full row (normal) rank, we obtain that $\bar{T}_{0} T_{0}^{-1}=\left[\begin{array}{cc}* & * \\ 0 & J_{1}\end{array}\right]$, which at once implies that 
$J_{1}(\delta)$ is invertible over $\mathbb{R}[\delta]$. This applied to (4) gives (6). Now, let $N_{k}$ and $\bar{N}_{k}$ be generated with the different set of matrices $\left\{T_{0}, \ldots, T_{k-1}\right\}$ and $\left\{\bar{T}_{0}, \ldots, \bar{T}_{k-1}\right\}$, respectively, and suppose that $\bar{N}_{k}=D_{k} N_{k}$, for some invertible matrix $D_{k}$. Now by a straightforward calculation, we can verify that $N_{k+1}$ satisfies the following recursive algorithm

$$
Y_{k}\left[\begin{array}{cc}
N_{k} B & N_{k} A \\
D & C
\end{array}\right]=\left[\begin{array}{cc}
W_{k+1} & X_{k+1} \\
0 & N_{k+1}
\end{array}\right]
$$

where $Y_{k}$ is an invertible matrix over $\mathbb{R}[\delta]$ and $W_{k+1}$ has full row rank. Analogously, (7) is valid for $\bar{N}_{k+1}$ with some matrices $\bar{Y}_{k}$ and $\bar{W}_{k+1}$. Thus, we obtain that

$$
\begin{aligned}
Y_{k}\left[\begin{array}{cc}
D_{k}^{-1} & 0 \\
0 & I
\end{array}\right] \bar{Y}_{k}^{-1} \bar{Y}_{k}\left[\begin{array}{cc}
D_{k} N_{k} B & D_{k} N_{k} A \\
D & C
\end{array}\right] & =Y_{k}\left[\begin{array}{cc}
D_{k}^{-1} & 0 \\
0 & I
\end{array}\right] \bar{Y}_{k}^{-1}\left[\begin{array}{cc}
\bar{W}_{k+1} & \bar{X}_{k+1} \\
0 & \bar{N}_{k+1}
\end{array}\right] \\
& =\left[\begin{array}{cc}
W_{k+1} & X_{k+1} \\
0 & N_{k+1}
\end{array}\right]
\end{aligned}
$$

Therefrom, we deduce that there exists an invertible (over $\mathbb{R}[\delta]$ ) matrix $D_{k+1}$ such that $\bar{N}_{k+1}=$ $D_{k+1} N_{k+1}$. Therefore, $\bar{N}_{k+1}$ and $N_{k+1}$ have the same smith form, which yields that $\bar{M}_{k+1}$ be identical to $M_{k+1}$. Since $N_{1}=\Delta_{1}$, we have proved by induction that $\bar{M}_{i}=M_{i}$, for all $i \geq 1$.

Lemma 2. By using the notation $d_{j}^{k}(\boldsymbol{\delta})$ as the $j$-th determinant divisor of $M_{k}(\boldsymbol{\delta})$ (generated by (4)-(5)), we obtain $d_{j}^{k+1}(\boldsymbol{\delta}) \mid d_{j}^{k}(\boldsymbol{\delta})$, for every $j \leq \operatorname{rank}_{k}(\boldsymbol{\delta})$.

Proof. Firstly let us notice that by (5), we obtain the following identity,

$$
\left[\begin{array}{cc}
U_{k} & 0 \\
0 & I
\end{array}\right] N_{k+1} V_{k}=\left[\begin{array}{c}
M_{k} \\
0 \\
\Delta_{k+1} V_{k}
\end{array}\right] \triangleq H_{k+1}
$$

So the invariant factors of $N_{k+1}$ are the same of those of the matrix in the right hand side of $H_{k}$. Thus, by definition $d_{1}^{k+1}(\delta)$ is the ged of all $1 \times 1$ minors of $H_{k+1}(\delta)$. Then, $d_{1}^{k+1}(\delta)$ divides every element of $H_{k+1}(\boldsymbol{\delta})$. Therefore, $d_{1}^{k+1}(\boldsymbol{\delta})$ divides $d_{1}^{k}(\boldsymbol{\delta})$. In general, $d_{i}^{k+1}(\boldsymbol{\delta})$ divides every $i \times i$ minor of $H_{k+1}(\delta)$. In what follows, let us assume that $j \leq \operatorname{rankM}_{k}(\delta)$. 
Now, for any matrix the product of the first $j$ invariant factors is equal to its $j$-th determinant divisor. Indeed, $\prod_{i=1}^{j} \psi_{i}=\frac{d_{1}}{d_{0}} \times \frac{d_{2}}{d_{1}} \times \cdots \times \frac{d_{j}}{d_{j-1}}=d_{j}$. Thus, by (5), $d_{j}^{k}(\delta)$ is a $j \times j$ minor of $N_{k+1}(\delta)$. Hence, we easily obtain that $d_{j}^{k+1}(\delta) \mid d_{j}^{k}(\boldsymbol{\delta})$.

Lemma 3. $M_{k+1}(\delta)=M_{k}(\delta)$ if, and only if, $\Delta_{k+1}(\delta)=P(\delta) N_{k}(\delta)$ for some matrix $P(\delta)$.

Proof. Sufficiency: If $\Delta_{k+1}(\boldsymbol{\delta})=P(\boldsymbol{\delta}) N_{k}(\boldsymbol{\delta})$, then

$$
N_{k+1}(\boldsymbol{\delta})=\left(\begin{array}{cc}
I & 0 \\
P(\boldsymbol{\delta}) & I
\end{array}\right)\left(\begin{array}{c}
N_{k}(\boldsymbol{\delta}) \\
0
\end{array}\right)
$$

This implies that $M_{k+1}(\delta)=M_{k}(\delta)$.

Necessity: From (8), we have that the invariant factors of $M_{k+1}(\delta)$ are equal to the invariant factors of $H_{k+1}$. Thus, if $M_{k+1}(\delta)=M_{k}(\delta)$, using the fact that $d_{1}^{k+1}=d_{1}^{k}$ divides every element of $H_{k+1}$, then we can verify that the first column of $\Delta_{k+1} V_{k}$ can be reduced to zero by premultiplying an invertible matrix to $H_{k+1}(\delta)$. We can apply the same procedure to the new obtained matrix, but applied to the second column. Thus, we achieve that every element below the second invariant factor can be made zero by a premultiplication with an unitary matrix, and so on for all the new obtained matrices, until we achieve to show that there exist an invertible matrix $U$ satisfying the identity

$$
U H_{k+1}=U\left[\begin{array}{c}
M_{k}(\boldsymbol{\delta}) \\
0 \\
\Delta_{k+1}(\boldsymbol{\delta}) V_{k}(\boldsymbol{\delta})
\end{array}\right]=\left[\begin{array}{c}
M_{k}(\boldsymbol{\delta}) \\
0 \\
0
\end{array}\right]
$$

The previous identity implies that

$$
\Delta_{k+1}(\delta) V_{k}(\boldsymbol{\delta})=L(\delta)\left[\begin{array}{c}
M_{k}(\boldsymbol{\delta}) \\
0
\end{array}\right]
$$

for a polynomial matrix $L(\delta)$. Thus, we have that

$$
\Delta_{k+1}(\boldsymbol{\delta}) V_{k}(\boldsymbol{\delta})=L(\boldsymbol{\delta}) U_{k}(\boldsymbol{\delta}) N_{k}(\boldsymbol{\delta}) V_{k}(\boldsymbol{\delta})
$$

That is $\Delta_{k+1}(\boldsymbol{\delta})=P(\boldsymbol{\delta}) N_{k}(\boldsymbol{\delta}),\left(P(\boldsymbol{\delta})=L(\boldsymbol{\delta}) U_{k}(\boldsymbol{\delta})\right)$. 
Theorem 1. If $M_{k+1}(\delta)=M_{k}(\boldsymbol{\delta})$, then $M_{k+i}(\delta)=M_{k}(\delta)$ for all $i \geq 0$.

Proof. As we have seen, $N_{k+1}$, for $k \geq 0$ satisfies the following recursive algorithm

$$
Y_{k}\left[\begin{array}{cc}
N_{k} B & N_{k} A \\
D & C
\end{array}\right]=\left[\begin{array}{cc}
W_{k+1} & X_{k+1} \\
0 & N_{k+1}
\end{array}\right]
$$

where $Y_{k}$ is an invertible matrix over $\mathbb{R}[\delta]$ and $W_{k+1}$ has full row rank. Now, assuming that $M_{k+1}=M_{k}$, according to Lemma 3, there exist a matrix $P$ such that $\Delta_{k+1}=P N_{k}$. Thus, let $\Psi$ be an invertible matrix over $\mathbb{R}[\boldsymbol{\delta}]$ (used to rearrange terms) chosen so that

$$
\Psi\left[\begin{array}{cc}
N_{k+1} B & N_{k+1} A \\
D & C
\end{array}\right]=\left[\begin{array}{cc}
N_{k} B & N_{k} A \\
D & C \\
P N_{k} B & P N_{k} A
\end{array}\right]
$$

The previous identity and (9) yield

$$
\left.\left[\begin{array}{cc}
Y_{k} & 0 \\
-P & 0
\end{array}\right] \quad I\right] \Psi\left[\begin{array}{cc}
N_{k+1} B & N_{k+1} A \\
D & C
\end{array}\right]=\left[\begin{array}{cc}
W_{k+1} & X_{k+1} \\
0 & N_{k+1} \\
0 & 0
\end{array}\right]
$$

Thus, we can define $\tilde{Y}_{k+1}$ and $\tilde{N}_{k+1}$ as follows,

$$
\begin{aligned}
& \left.\tilde{Y}_{k+1} \triangleq\left[\begin{array}{cc}
Y_{k} & 0 \\
-P & 0
\end{array}\right] \Psi\right] \Psi \\
& \tilde{N}_{k+2}=\left[\begin{array}{c}
N_{k+1} \\
0
\end{array}\right]
\end{aligned}
$$

where the zero vector of $\tilde{N}_{k+2}$ has the same number of rows as $P$ has. Then, we have just proved that $\tilde{N}_{k+2}$ satisfies the equation

$$
\tilde{Y}_{k+1}\left[\begin{array}{cc}
N_{k+1} B & N_{k+1} A \\
D & C
\end{array}\right]=\left[\begin{array}{cc}
W_{k+1} & X_{k+1} \\
0 & \tilde{N}_{k+2}
\end{array}\right]
$$

Moreover, according to (9) $N_{k+2}$ satisfies

$$
Y_{k+1}\left[\begin{array}{cc}
N_{k+1} B & N_{k+1} A \\
D & C
\end{array}\right]=\left[\begin{array}{cc}
W_{k+2} & X_{k+2} \\
0 & N_{k+2}
\end{array}\right]
$$


where $Y_{k+1}$ is invertible over $\mathbb{R}[\delta]$ and $W_{k+2}$ has full row rank. Thus, we can use similar arguments to those used to prove the identity (6) to prove that $N_{k+2}=R \tilde{N}_{k+2}$, with $R$ being an invertible matrix over $\mathbb{R}[\delta]$. Thereby, the invariant factors of $N_{k+2}$ are equal to those of $\tilde{N}_{k+2}$, which in fact has the same invariant factors as $N_{k+1}$, because of the definition of $\tilde{N}_{k+2}$. Thus, $N_{k+2}$ and $N_{k+1}$ have exactly the same invariant factors. Moreover, since by the definition of $M_{k+1}, N_{k+1}$ and $M_{k+1}$ have the same invariant factors, and by assumption $M_{k+1}=M_{k}$, we have that the invariant factors of $N_{k+2}$ are exactly those of $M_{k}$. Finally, again since $M_{k+2}$ has the same invariant factors as $N_{k+2}$, we have proved that $M_{k+2}$ and $M_{k}$ have identical invariant factors. Then, by the definition of $\left\{M_{j}\right\}$ matrices, we arrive to the identity $M_{k+2}=M_{k}$. Following exactly the same procedure, by induction, we prove that $M_{k+i}=M_{k}$ for all $i \geq 0$.

Theorem 2. After a finite number of steps, let say $k^{*}$, the algorithm (4)-(5) converges, i.e., there exists a least integer $k^{*}$ such that $M_{k^{*}+1}(\delta)=M_{k^{*}}(\delta)$. Furthermore, $k^{*}$ is invariant of the choice of $\left\{T_{k}\right\},\left\{U_{k}\right\}$, and $\left\{V_{k}\right\}$ matrices used in (4)-(5).

Proof. Since, by (5), $\operatorname{rank}_{k+1}(\delta) \geq \operatorname{rankM}_{k}(\boldsymbol{\delta})$, and $\operatorname{rank}\left(M_{k}(\delta)\right) \leq n$ (for all $k$ ), then after a finite number of steps, let say $\bar{k}$, we obtain that

$$
\operatorname{rank}_{\bar{k}+i}(\delta)=\operatorname{rankM}_{\bar{k}}(\delta), \text { for all } i \geq 0
$$

By Lemma $2, d_{j}^{k+1}$ divides $d_{j}^{k}$ (this is true for every $k \geq 0$ ), and also we know that $d_{j}^{k}$ divides $d_{j+1}^{k}$. Hence, we have that $\operatorname{deg} d_{j}^{\bar{k}+1} \leq \operatorname{deg} d_{j}^{\bar{k}}$ and $\operatorname{deg} d_{j}^{\bar{k}} \leq \operatorname{deg} d_{j+1}^{\bar{k}}$. Let us define $\alpha(k)=$ $\sum_{j=1}^{r_{k}} \operatorname{deg} d_{j}^{k},\left(r_{k} \triangleq \operatorname{rank}_{k}\right)$, then (since $\left.r_{\bar{k}}=r_{\bar{k}+1}\right)$ we readily obtain the inequality

$$
0 \leq \alpha(k+1) \leq \alpha(k) \text { for all } k \geq \bar{k}
$$

Previous inequality means that there exists $\tilde{k} \geq \bar{k}$ such that $\alpha(\tilde{k}+1)=\alpha(\tilde{k})$. This implies that $\operatorname{deg} d_{j}^{\tilde{k}}=\operatorname{deg} d_{j}^{\tilde{k}+1}$ for every $j \leq r_{\tilde{k}} \operatorname{since} \operatorname{deg} d_{j}^{\tilde{k}+1} \leq \operatorname{deg} d_{j}^{\tilde{k}}$. But, if $\operatorname{deg} d_{j}^{\tilde{k}}=\operatorname{deg} d_{j}^{\tilde{k}+1}$, then $d_{j}^{\tilde{k}}=d_{j}^{\tilde{k}+1}$ because of $d_{j}^{\tilde{k}}$ is the product of monic polynomials. Therefore, since $d_{j+1}^{\tilde{k}}=d_{j}^{\tilde{k}} \psi_{j+1}^{\tilde{k}}$, then $\alpha(\tilde{k}+1)=\alpha(\tilde{k})$ implies that $\psi_{j+1}^{\tilde{k}+1}=\psi_{j+1}^{\tilde{k}}$, which in turn means that $M_{\tilde{k}+1}=M_{\tilde{k}}$. Thus, $k^{*}$ is the least integer for which $M_{k^{*}+1}=M_{k^{*}}$ is satisfied. The invariance of $k^{*}$ from the choices of $\left\{T_{k}\right\},\left\{U_{k}\right\}$, and $\left\{V_{k}\right\}$ matrices comes directly from Lemma 1 . 
Remark 3. In fact the proof of Theorem 2 says us a little more. It says that if rank $M_{k+1}=\operatorname{rank}_{k}$, then either $M_{k+\alpha(k)+1}=M_{k+\alpha(k)}$ or rank $M_{k+\alpha(k)+1}>\operatorname{rank} M_{k+\alpha(k)}$. This gives a way to have an upper estimation of how many steps will pass before the rank of $M_{k}$ does change, if it does.

Remark 4. We would like to emphasize that rankM $M_{k+1}=$ rankM $M_{k}$ does not imply that $M_{k+1}=M_{k}$. Indeed, let see the following simple example,

$$
\begin{aligned}
& \dot{x}_{1}(t)=x_{2}(t), \dot{x}_{2}(t)=x_{3}(t), \dot{x}_{3}(t)=x_{4}(t), \dot{x}_{4}(t)=x_{2}+x_{2}(t-h), \\
& y_{1}(t)=x_{1}(t) \text { and } y_{2}(t)=x_{4}(t-h)
\end{aligned}
$$

For this example, rankM $M_{3}=$ rankM 4. However, $M_{3}$ is invertible over $\mathbb{R}\left[\delta, \delta^{-1}\right]$, but is not invertible over $\mathbb{R}[\delta]$. Nevertheless, it is easy to verify that $M_{4}$ is invertible over $\mathbb{R}[\delta]$.

Next corollary is a direct consequence of the previous results.

Corollary 1. Let $k^{*}$ be the least integer such that $M_{k^{*}+1}=M_{k^{*}}$, then for all $i \geq 0, M_{k^{*}+i}=M_{k^{*}}$.

\section{State Reconstruction}

From now it is possible to give sufficient observability conditions. In the way to arrive to such conditions we will draw the connection of the recursive algorithm used to obtain $\Delta_{k}(\delta)$ and a way that may be used for the reconstruction of the state vector. Let us define $\hat{y}^{1}(t)=T_{0}(\delta)\left[\begin{array}{c}0 \\ y(t)\end{array}\right]$. Thus, from (2) and (4) we obtain

$$
\hat{y}^{1}(t) \triangleq\left[\begin{array}{c}
\hat{y}_{1}^{1}(t) \\
\hat{y}_{2}^{1}(t)
\end{array}\right]=\left[\begin{array}{c}
G_{1}(\boldsymbol{\delta}) x(t)+F_{1}(\boldsymbol{\delta}) w(t) \\
\Delta_{1}(\boldsymbol{\delta}) x(t)
\end{array}\right]
$$

From here, we will define a chain of vectors $\left\{\hat{y}^{i}(t)\right\}$. Thus, $\hat{y}_{1}^{i}(t)$ and $\hat{y}_{2}^{i}(t)$ will be the upper and lower subvectors of $\hat{y}^{i}(t)$, respectively, whose dimension will be implicitly defined.

We have already obtained a virtual output $\hat{y}_{2}^{1}(t)$ without the influence of the unknown input $w(t)$. Let us define $\alpha_{1}=\operatorname{deg} \Delta_{1}(\delta)$, then, for $t \geq \alpha_{1} h, \hat{y}_{2}^{1}(t)$ is differentiable. Hence, according to (11) and (2), we obtain the following equation,

$$
\frac{d}{d t} \hat{y}_{2}^{1}(t)=\frac{d}{d t} \Delta_{1}(\delta) x(t)=\Delta_{1}(\boldsymbol{\delta}) A(\boldsymbol{\delta}) x(t)+\Delta_{1}(\boldsymbol{\delta}) B(\boldsymbol{\delta}) w(t), t \geq \alpha_{1} h
$$


Now, let us generate an extended vector $\xi_{1}(t)$, defined as follows

$$
\xi_{1}(t) \triangleq\left[\begin{array}{c}
\frac{d}{d t} \hat{y}_{2}^{1}(t) \\
\hat{y}_{1}^{1}(t)
\end{array}\right]
$$

Again, we define $\hat{y}^{2}(t)=T_{1}(\delta) \xi_{1}(t), t \geq \alpha_{1} h$. In view of (4) and (12), we obtain the following identity,

$$
\hat{y}^{2}(t) \triangleq\left[\begin{array}{c}
\hat{y}_{1}^{2}(t) \\
\hat{y}_{2}^{2}(t)
\end{array}\right]=\left[\begin{array}{c}
G_{2}(\delta) x(t)+F_{2}(\boldsymbol{\delta}) w(t) \\
\Delta_{2}(\boldsymbol{\delta}) x(t)
\end{array}\right]
$$

Differentiation of $\hat{y}_{2}^{2}(t)$ gives, for $t \geq h \max \left(\alpha_{1}, \alpha_{2}\right)$ (where $\alpha_{2}=\operatorname{deg} \Delta_{2}(\delta)$ )

$$
\frac{d}{d t} \hat{y}_{2}^{2}(t)=\Delta_{2}(\delta) A(\delta) x(t)+\Delta_{2}(\delta) B(\delta) w(t)
$$

In the same manner, we define a second extended vector $\xi_{2}(t)$ :

$$
\xi_{2}(t)=\left[\begin{array}{c}
\frac{d}{d t} \hat{y}_{2}^{2}(t) \\
\hat{y}_{1}^{2}(t)
\end{array}\right]
$$

After defining $\hat{y}_{3}(t) \triangleq T_{2}(\delta) \xi_{2}(t)$, we obtain the identities $\hat{y}_{1}^{3}(t)=G_{3}(\delta) x(t)+F_{3}(\delta) w(t)$ and $\hat{y}_{2}^{3}(t)=\Delta_{3}(\delta) x(t)$.

The previous procedure, allows us for writing the following expressions, for $k \geq 1$ and $t \geq$ $h \max _{1<i<k-1} \operatorname{deg} \Delta_{i}(\boldsymbol{\delta})$,

$$
\begin{aligned}
& \xi_{k}(t) \triangleq\left[\begin{array}{c}
\frac{d}{d t} \hat{y}_{2}^{k}(t) \\
\hat{y}_{1}^{k}(t)
\end{array}\right], \quad \xi_{0}(t) \triangleq\left[\begin{array}{c}
0 \\
y(t)
\end{array}\right] \\
& \hat{y}^{k}(t) \triangleq\left[\begin{array}{c}
\hat{y}_{1}^{k}(t) \\
\hat{y}_{2}^{k}(t)
\end{array}\right] \triangleq T_{k-1}(\delta) \xi_{k-1}(t) \\
& \hat{y}_{2}^{k}(t)=\Delta_{k}(\delta) x(t)
\end{aligned}
$$

Thus, we define $Y(t)$ as

$$
Y(t) \triangleq\left[\begin{array}{c}
\hat{y}_{2}^{1}(t) \\
\hat{y}_{2}^{2}(t) \\
\vdots \\
\hat{y}_{2}^{k^{*}}(t)
\end{array}\right]
$$


With the definition $t^{*}=h \max _{1 \leq i \leq k^{*}-1} \operatorname{deg} \Delta_{i}(\delta)$, by (13) we have that

$$
Y(t)=N_{k^{*}}(\delta) x(t) \text { for all } t \geq t^{*}
$$

Let us introduce the forward time-shift operator as $\delta^{-1}: x(t) \rightarrow x(t+h)$, which is in fact the inverse of $\delta$. [I guess that you just replaced the old $\Delta$, but forgot to change this part!!] We also recall that the Euclidian domain $\mathbb{R}\left[\delta, \delta^{-1}\right]$ contains the Euclidian domain $\mathbb{R}[\delta]$. Thus, the following proposition is an obvious consequence of properties of $\mathbb{R}\left[\delta, \delta^{-1}\right]$.

Proposition 1. $M_{k^{*}}(\delta)$ matrix has an inverse on $\mathbb{R}\left[\delta, \delta^{-1}\right]$ if, and only if, $M_{k^{*}}(\delta)$ has $n$ invariant factors, all of them of the form $a \delta^{j}$, with $a \in \mathbb{R}_{\neq 0}$ and $j \in \mathbb{N}_{0}$.

Proof. All units (elements that have an inverse) of $\mathbb{R}\left[\delta, \delta^{-1}\right]$ are of the form $a \delta^{j}$ where $a$ belongs to $\mathbb{R}_{\neq 0}$ and $j$ is an integer. Thus, since $M_{k^{*}}(\delta)$ has an inverse on $\mathbb{R}\left[\delta, \delta^{-1}\right]$ if, and only if, $\operatorname{det} M_{k^{*}}(\delta)$ is a unit on $\mathbb{R}\left[\delta, \delta^{-1}\right]$, then $M_{k^{*}}(\delta)$ should have $n$ invariant factors all of them of the form described $a \delta^{j}$, with $a$ in $\mathbb{R}_{\neq 0}$ and $j$ a nonnegative integer (due to each invariant factor of $M_{k^{*}}(\boldsymbol{\delta})$ is in $\left.\mathbb{R}[\boldsymbol{\delta}]\right)$.

Let us define $t_{1}^{*}$ as follows

$$
t_{1}^{*}=h \times \operatorname{deg}\left(\left[\begin{array}{cc}
V_{k^{*}} M_{k^{*}}^{-1} & 0
\end{array}\right] U_{k^{*}}\right)+t^{*}
$$

I think there should be a $h$ in front of deg...

Theorem 3. The state vector $x(t)$ can be reconstructed in finite time, for any $t>t_{1}^{*}$, if $M_{k^{*}}(\delta)$ has $n$ invariant factors of the form $a \delta^{j}$, where $a \in \mathbb{R}_{\neq 0}, j \in \mathbb{N}_{0}$. The formula to reconstruct $x(t)$ is expressed as

$$
x(t)=\left[\begin{array}{ll}
V_{k^{*}} M_{k^{*}}^{-1} & 0
\end{array}\right] U_{k^{*}} Y(t), t>t_{1}^{*}
$$

Furthermore, the $i$-th entry of $x(t)$ is given by an expression of the form:

$$
x_{i}(t)=\sum_{k, j} q_{k, j} y_{k}^{(j)}(t)
$$

where $y_{k}^{(i)}(t)$ is the $i$-th derivative of the $k$-th entry of the vector $y(t)$ and every $q_{j, k}^{i}$ is a nonzero element of $\mathbb{R}\left[\delta, \delta^{-1}\right]$. 
Proof. We have seen in Proposition 1 that the hypothesis of the theorem implies that $M_{k^{*}}(\delta)$ is invertible over $\mathbb{R}\left[\delta, \delta^{-1}\right]$. Thus, by (14), we obtain the identity (16) for all $t \geq t_{1}^{*}$.

The expression in (17) can be easily deduced by taking into account $Y(t)=N_{k^{*}}(\delta) x(t)$ (for all $t \geq t^{*}$ ) and (13). Indeed, every element of $\hat{y}^{1}(t)$ is just a linear combination of the entries of $y(t)$, and so the entries of $\xi_{1}$ are linear combinations of elements of $y(t)$ and derivatives of entries of $y(t)$, and so on.

Remark 5. For the trivial case $h=0$, the condition that $M_{k^{*}}$ is invertible over $\mathbb{R}$ is also a necessary condition for the system to be UIO (known it as strong observability, see, e.g. Molinari (1976) and Trentelman et al. (2001)).

Remark 6. For the case when $B(\delta)=0$ and $D(\delta)=0$, the condition that $M_{k^{*}}$ be invertible over $\mathbb{R}[\delta]$ is equivalent to the condition that $\left((C(\delta))^{T},(C(\delta) A(\delta))^{T}, \cdots,\left(C(\delta) A^{n-1}(\delta)\right)^{T}\right)^{T}$ be left invertible over $\mathbb{R}[\delta]$. This condition is known as strong observability also (see, Lee and Olbrot (1981)).

In view of the previous two remarks, we suggest a definition of strong observability (as a generalization) for the systems considered in this work. We consider $\mathbb{R}\left[\delta, \delta^{-1}\right]$ as the ring over which the matrix (given below) may be invertible, this allows to have a less restrictive characterization of the observability.

Definition 4. System (1) is said to be strongly observable (SO) if, and only if, $M_{k^{*}}$ is invertible over $\mathbb{R}\left[\delta, \delta^{-1}\right]$, i.e. iff $M_{k^{*}}$ has $n$ invariant factors all of them of the form a $\delta^{j}\left(a \in \mathbb{R}_{\neq 0}, j \in \mathbb{N}_{0}\right)$.

Now, we can deduce easily sufficient conditions for the UIO, BUIO and FUIO.

Corollary 2. If system (1) is SO then it is UIO on $\left[t_{1}^{*}, t_{2}\right]$, for all $t_{2}>t_{1}^{*}$.

Proof. Let $r_{1}$ and $r_{2}$ be respectively the greatest and the least of powers of the polynomials $q_{j, k}^{i}$ for all $i \in \overline{1, n}$ and every $j \in \overline{1, s(i)}$ and $k \in \overline{1, r_{j}^{i}}$. Since (17) is valid for $t \geq t_{1}^{*}$, then $y(t)=0$ on $\left[t_{1}^{*}-r_{1} h, t_{1}^{*}+r_{2} h\right]$ yields the identity $q_{j, k}^{i} y_{i}^{(j)}\left(t_{1}^{*}\right)=0$ for all possible $i, j, k$, which in turn implies that $x\left(t_{1}^{*}\right)=0$. Likewise, for all $t_{2}>t_{1}^{*}$, we see that $y(t)=0$ on $\left[t_{1}^{*}-r_{1} h, t_{2}+r_{2} h\right]$ implies $x(t)=0$ on $\left[t_{1}^{*}, t_{2}\right]$, which proves the theorem. 
Corollary 3. If system (1) is $S O$ and, for all $\overline{i \in 1, n}$, every polynomial $q_{j, i}$ of (17) belongs to $\mathbb{R}[\delta]$, then (1) is BUIO on $\left[t_{1}^{*}, t_{2}\right]$, for all $t_{2}>t_{1}^{*}$.

Proof. Since (16) is valid for a $t_{1}^{*} \geq 0$, then in case every $q_{j, i} \in \mathbb{R}[\delta]$, we obtain that for any $t_{2} \geq t_{1}^{*}$, the identity $y(t)=0$ on $\left[t_{1}^{*}-\bar{k} h, t_{2}\right]\left(\bar{k}\right.$ being the biggest degree of all polynomials $\left.q_{j, i}\right)$ implies that $x\left(t_{2}\right)=0$.

Proposition 2. Let us assume that system (1) is SO. Then every polynomial $q_{j, i}$ in (17) belongs to $\mathbb{R}[\delta]$ if, and only if, $\operatorname{det} M_{k^{*}}=1$.

Proof. Let us define the set $\Omega$ as the set of polynomials of the form

$$
\sum_{k, i} p_{k, i} y_{k}^{(i)}(t), k, i \geq 1
$$

where $p_{k, i}$ are nonzero polynomials of $\mathbb{R}[\delta]$ and at least one of them belongs to $\mathbb{R}_{\neq 0}$. Let us suppose that every element of the vector $Y(t)$ belongs to $\Omega$. Upon this, let $U$ be invertible over $\mathbb{R}[\delta]$, then every element of the vector $U Y(t)$ is also in $\Omega$. This is true since every row (and column) of $U$ has a (nonzero) element of the form $a+\sum_{k} b_{k} \delta^{k}$, with $a \in \mathbb{R}_{\neq 0}$ and every $k$ is greater than 0 . Thus, again every element of the vector $\left[\begin{array}{ll}I & 0\end{array}\right] U_{k^{*}} Y(t)$ belongs to $\Omega$. Therefore, we see that the $j$-th element of $M_{k^{*}}\left[\begin{array}{ll}I & 0\end{array}\right] U_{k^{*}} Y(t)$ is of the form $\sum_{k, i} q_{k, i} y_{k}^{(i)}(t)$ with all $q_{k, i} \in \mathbb{R}[\delta]$, if, and only if, the element of the $j$-th row and $j$-th column of $M_{k^{*}}$ is equal to 1 (this is due to the fact that $M_{k^{*}}$ is a diagonal matrix and all its nonzero elements are monic). Therefore, to prove the proposition we need to prove that every element of $Y(t)$ is within $\Omega$.

Let $\hat{y}_{2}^{1}(t)$ be as in (13). It is easy to check that every element of this vector belongs to $\Omega$. This is true because of each entry of $\hat{y}_{2}^{1}(t)$ is the product of a row of $T_{0}$ (which is invertible over $\mathbb{R}[\delta]$ ) and the vector $\left[\begin{array}{ll}0 & y^{T}\end{array}\right]^{T}$. With the same argument we have that $\frac{d}{d t} \hat{y}_{2}^{1}(t)$ has all its elements in $\Omega$. Now, as for $\hat{y}_{2}^{2}(t)$, we have that it is the product of a row of $T_{1}$ (which is invertible over $\mathbb{R}[\boldsymbol{\delta}]$ ) and the vector formed by $\hat{y}_{1}^{1}(t)$ and $\frac{d}{d t} \hat{y}_{2}^{1}(t)$. So, again every element of $\hat{y}_{2}^{2}(t)$ belongs to $\Omega$. By following the same process, we conclude that each element of every vector $\hat{y}_{2}^{i}(t)(i \geq 1)$ belongs to the set $\Omega$. Thus since $Y(t)$ is the arrange of those vector, then every element of $Y(t)$ belongs to $\Omega$. The proposition has been proved. 
Corollary 4. Assume that system (1) is SO. Then it is BUIO on $\left[t_{1}^{*}, t_{2}\right]$, for all $t_{2}>t_{1}^{*}$, if, and only if, $\operatorname{det} M_{k^{*}}=1$.

Proof. The proof is straightforward by Corollary 3 and Proposition 2.

As for FUIO, we have the following corollary which characterized it, provided (1) is SO.

Corollary 5. If system (1) is $S O$ and, for all $\overline{i \in 1, n}$, every polynomial $q_{j, i}$ of (17) belongs to $\mathbb{R}\left[\delta^{-1}\right]$, then (1) is FUIO on $\left[t_{1}^{*}, t_{2}\right]$, for all $t_{2}>t_{1}^{*}$.

Proof. If every polynomial $q_{j, i}$ belongs to $\mathbb{R}\left[\delta^{-1}\right]$, then, for every $t_{2} \geq t_{1}^{*}$, the identity $y(t)=0$ on $\left[t_{2}, t_{1}^{*}+k h\right]$ implies $x\left(t_{2}\right)=0\left(\bar{k}\right.$ being the biggest degree of all polynomials $\left.q_{j, i}\right)$.

\section{Examples}

Example 1. Let us consider the following example:

$$
\begin{aligned}
& A(\delta)=\left(\begin{array}{ccccc}
1 & \delta & \delta & 0 & 0 \\
-\delta^{2} & 0 & \delta & 0 & -\delta \\
\delta & 1 & -\delta^{2} & -1+\delta & 1-\delta+\delta^{3} \\
0 & 0 & -1 & 0 & 0 \\
\delta-\delta^{2} & 0 & -1+\delta & 2 & 1-\delta
\end{array}\right), B(\delta)=\left(\begin{array}{cc}
-\delta & 0 \\
0 & -\delta \\
1+\delta & 1 \\
1 & 0 \\
0 & 1-\delta
\end{array}\right) \\
& C(\boldsymbol{\delta})=\left(\begin{array}{lllll}
1 & 0 & 0 & 0 & 0 \\
0 & 1 & 0 & 0 & 0 \\
0 & 0 & 0 & 0 & \delta
\end{array}\right), D(\delta)=\left(\begin{array}{cc}
0 & 0 \\
1 & 0 \\
-1+\delta & 0
\end{array}\right)
\end{aligned}
$$

According to (4), we have that

$$
T_{0}=\left[\begin{array}{cccc}
0 & 0 & 1 & 0 \\
0 & 1 & 0 & 0 \\
1 & 0 & 0 & 0 \\
0 & 0 & 1-\delta & 1
\end{array}\right], \quad \Delta_{1}=\left[\begin{array}{ccccc}
1 & 0 & 0 & 0 & 0 \\
0 & 0 & 0 & 0 & 0 \\
0 & 1-\delta & 0 & 0 & \delta
\end{array}\right],
$$

Thus, in view of (5), we obtain

$$
M_{1}=\left[\begin{array}{ccccc}
1 & 0 & 0 & 0 & 0 \\
0 & 1 & 0 & 0 & 0
\end{array}\right]
$$


Following with the procedure we obtain the matrices

$$
T_{1}=\left[\begin{array}{cccc}
0 & 0 & 0 & 1 \\
0 & 1 & 0 & 0 \\
0 & 0 & 1 & 0 \\
1 & 0 & 0 & \delta
\end{array}\right], \Delta_{2}=\left[\begin{array}{ccccc}
0 & 0 & 0 & 0 & 0 \\
0 & 0 & 0 & 2 \delta & 0 \\
1 & 2 \delta & \delta & 0 & 0
\end{array}\right], M_{2}=\left[\begin{array}{ccccc}
1 & 0 & 0 & 0 & 0 \\
0 & 1 & 0 & 0 & 0 \\
0 & 0 & \delta & 0 & 0 \\
0 & 0 & 0 & \delta & 0
\end{array}\right]
$$

and

$$
T_{2}=\left[\begin{array}{cccc}
0 & 0 & 0 & 1 \\
0 & 0 & -\frac{1}{2} & \frac{1}{2} \delta^{2} \\
0 & \frac{1}{2} & 0 & -\delta \\
1 & 0 & 0 & 0
\end{array}\right], \Delta_{3}=\left[\begin{array}{ccccc}
0 & -\delta & -\delta & 0 & 0 \\
0 & 0 & 0 & 0 & 0
\end{array}\right], M_{3}=M_{4}=\operatorname{diag}\left(1,1, \delta, \delta, \delta^{2}\right)
$$

Thus, in this case $k^{*}=3$, and the system is UIO since all the invariant factors of $M_{3}$ belongs to $\mathbb{R}\left[\delta, \delta^{-1}\right]$. Explicitly, we have that the state vector can be expressed as

$$
\begin{aligned}
x_{1}= & y_{1} \\
x_{2}= & -\delta^{-1} y_{1}+\delta^{-1} \dot{y}_{1}+\frac{1}{2}\left(1-\delta^{-1}\right) \dot{y}_{2}-\frac{1}{2} \delta^{-1} \dot{y}_{3} \\
x_{3}= & \delta^{-1} y_{1}+y_{2}-\delta^{-1} \dot{y}_{1}-\frac{1}{2}\left(1-\delta^{-1}\right) \dot{y}_{2}+\frac{1}{2} \delta^{-1} \dot{y}_{3} \\
& -\frac{1}{2}\left(1-\delta^{-1}\right) \ddot{y}_{2}+\frac{1}{2} \delta^{-1} \ddot{y}_{3} \\
x_{4}= & \frac{1}{2}\left(1-\delta^{-1}\right) \dot{y}_{2}-\frac{1}{2} \delta^{-1} \dot{y}_{3} \\
x_{5}= & \left(\delta^{-2}-\delta^{-1}\right) y_{1}+\left(\delta^{-1}-1\right) y_{2}+\delta^{-1} y_{3}+\left(\delta^{-1}-\delta^{-2}\right) \dot{y}_{1} \\
& +\frac{1}{2}\left(1-\delta^{-1}\right)^{2} \dot{y}_{2}+\frac{1}{2}\left(\delta^{-2}-\delta^{-1}\right) \dot{y}_{3}
\end{aligned}
$$

Therefore, according to Corollary 5, the system is FUIO.

Example 2. Now, let us consider that the matrices of the system (2) are the following,

$$
\begin{aligned}
& A=\left(\begin{array}{ccc}
0 & -1 & 1 \\
-1 & \delta & 0 \\
1 & 0 & 1
\end{array}\right), B=\left(\begin{array}{ll}
0 & 0 \\
0 & 0 \\
0 & \delta
\end{array}\right) \\
& C=\left(\begin{array}{lll}
\delta & 0 & 0 \\
0 & 1 & 0
\end{array}\right), D=\left(\begin{array}{cc}
-2 \delta & 0 \\
0 & 0
\end{array}\right)
\end{aligned}
$$


In this example, we have that $M_{1}$ has an invariant factor equal to $1, M_{2}$ has the invariant factors $\{1,1\}$, and the invariant factors of $M_{3}$ are $\{1,1,1\}$. Hence the system is $S O$ and furthermore, it is BUIO also, that is the state vector can be reconstructed using actual and past values of the system output. Indeed, it is easy to verify that the state variables can be expressed as

$$
x_{1}(t)=-\dot{y}_{2}+\delta y_{2}, x_{2}(t)=y_{2}, \text { and } x_{3}=-\ddot{y}_{2}+\delta \dot{y}_{2}+y_{2}
$$

Example 3. Finally let us consider the following example:

$$
A=\left[\begin{array}{lll}
0 & 1 & 0 \\
0 & \delta & 1 \\
\delta & 0 & 0
\end{array}\right], B=0, C=\left[\begin{array}{lll}
1 & 0 & 0 \\
0 & 1 & 0
\end{array}\right], D=\left[\begin{array}{l}
1 \\
\delta
\end{array}\right]
$$

In this case $M_{3}=\operatorname{diag}(1, \delta, \delta)$, so it is UIO, however, it is neither BUIO nor FUIO. As we can see, $x$ depends on past, actual, and future values of the system output:

$$
x_{1}=-y_{1}+\delta^{-1} \dot{y}_{2}, x_{2}=-\dot{y}_{1}+\delta^{-1} \ddot{y}_{2} \text {, and } x_{3}=-\delta y_{1}+\dot{y}_{2}
$$

Remark 7. For the computation of matrices used to arrive to $M_{k^{*}}$, we have used the Matlab software which includes symbolic tools of Maple. For instance to calculate the Smith form of a matrix $N$, we used the instruction maple( 'smith', $N, s$ ). For calculating the Hermite form: maple( 'smith', $N, s)$. Multiplication of matrices is done in the standard way.

\section{Conclusions}

In this paper we have proposed to tackle the observability of linear commensurable time delay systems with UI using three different definitions. Essentially, the first definition (UIO) deals with the possibility of reconstruct the state vector using output information (using past, actual, and/or future values), the second definition (BUIO) is related with the state reconstruction using just actual and past output information. And the third definition (FUIO) is about the state reconstruction using future values of the system output. We have given sufficient conditions allowing for the system to be UIO, BUIO, or FUIO, respectively. As for the conditions given for the UIO we have seen that the condition obtained includes the already known conditions for systems with delays 
without unknown inputs and for the case of linear systems with unknown inputs without delays. It should It would be interesting to define the cases were the obtained conditions are also necessary. Our conjecture is that $\mathrm{SO}$ is also a necessary condition if the aim is to expressed the state vector as a polynomial function of the system output and some of its derivatives.

\section{References}

\section{References}

Bhat, K., Koivo, H., 1976. Modal characterizations of controllability and observability in time delay systems. IEEE Transactions on Automatic Control 21 (2), 292- 293.

Brewer, J., Bunce, J., Vleck, F. V., 1986. Linear systems over commutative rings. Marcel Dekker, New York.

Conte, G., Perdon, A. M., Guidone-Peroli, G., December 2003. Unknown input observers for linear delay systems: a geometric approach. In: Proceedings of the 42nd IEEE Conference on Decision and Control. Maui, Hawaii USA, pp. 6054-6059.

Conte, G., Perdon, A. M., Moog, C. H., 2007. Applications of Time Delay Systems. Vol. 352 of Lecture Notes in Control and Information Sciences. Springer Berlin Heidelberg, Ch. Inversion and tracking problems for time delay linear systems, pp. 267-284.

Darouach, M., June 2006. Full order unknown inputs observers design for delay systems. In: 14th Mediterranean Conference on Control and Automation. Ancona, Italy, pp. 1-5.

Fattouh, A., Sename, O., Dion, J.-M., December 1999. An unknown input observer design for linear time-delay systems. In: 38th IEEE Conference on Decision and Control. Phoenix, AZ, U.S., pp. 4222-4227.

Fliess, M., Mounier, H., 1998. Controllability and observability of linear delay systems: an algebraic approach. ESAIM: Control, Optimisation and Calculus of Variations 3, 301-314.

Fu, Y.-M., Duan, G.-R., Song, S.-M., December 2004. Design of unknown input observer for linear time-delay systems. International Journal of Control, Automation, and Systems 2 (4), 530-535.

Kalman, R. E., Falb, P. L., Arbib, M. A., 1969. Topics in Mathematical System Theory. McGraw-Hill, New York.

Lee, E., Olbrot, A., June 1981. Observability and related structural results for linear hereditary systems. International Journal of Control 34 (6), 1061-1078.

Molinari, B., October 1976. A strong controllability and observability in linear multivariable control. IEEE Transactions on Automatic Control 21 (5), 761-764.

Moog, C., Castro-Linares, R., Velasco-Villa, M., Marque-Martinez, L. A., 2000. The disturbance decoupling problem for time-delay nonlinear systems. IEEE Transactions on Automatic Control 45 (2).

Olbrot, A. W., April 1981. Observability and observers for a class of linear systems with delays. IEEE Transactions on Automatic Control 26 (2), 513-517. 
Rabah, R., July 1995. On observability of linear delay systems with unknown inputs. In: 3rd IEEE Mediterranean Symposium on New Directions. Limassol, Cyprus.

Richard, J.-P., October 2003. Time-delay systems:an overview of some recent advances and open problems. Automatica 39 (10), 1667-1694.

Salamon, D., December 1980. Observers and duality between observation and state feedback for time delay systems. IEEE Transactions on Automatic Control 25 (6), 1187-1192.

Sename, O., 2001. New trends in design of observers for time-delay systems. Kybernetika 37 (4), 427-458.

Silverman, L., 1969. Inversion of multivariable linear systems. IEEE Transactions on Automatic Control 14 (3), 270276.

Sontag, E., 1976. Linear systems over commutative rings: A survey. Ricerche di Automatica 7, 1-34.

Trentelman, H., Stoorvogel, A., Hautus, M. L. J., 2001. Control Theory for Linear Systems. Communications and control engineering. Springer, New York, London.

Xia, X., Marquez, L., Zagalak, P., Moog, C., 2002. Analysis of nonlinear time-delay systems using modules over non-commutative rings. Automatica 38, 1549-1555.

Zheng, G., Barbot, J.-P., Boutat, D., Floquet, T., Richard, J.-P., 2011. On observation of time-delay systems with unknown inputs. IEEE Transactions on Automatic Control 56 (8), 1973-1978. 